\title{
Démarketing de la consommation d'électricité résidentielle avec les croyances relatives à la législation comme modérateur : une étude de cas sur la bande de Gaza, en Palestine ${ }^{1}$
}

\author{
Mohammed Z. Salem ${ }^{\mathrm{a}}$
}

RÉSUMÉ. La demande croissante d'électricité pose des défis dans certaines régions du monde. Ce constat est particulièrement vrai dans la bande de Gaza $(B d G)$ en Palestine, où la pénurie d'électricité est exacerbée par la forte croissance démographique et par le siège israélien de 10 ans ayant empêché la modernisation de la seule centrale électrique du territoire. Actuellement, la réduction de la consommation d'électricité est la seule solution réaliste pour pallier cette pénurie. Considérant que les ménages représentent une part importante de la consommation d'électricité, cet article examine dans quelle mesure leurs intentions de consommation peuvent être réduites en utilisant des stratégies de démarketing, donc de dissuasion. En outre, l'article teste l'efficacité de la réglementation à influencer les intentions de déconsommation résidentielle. Une étude empirique a été menée à l'aide d'une enquête en face à face pour collecter des données primaires afin de tester les hypothèses. Les résultats du questionnaire, distribué à 384 résidents sélectionnés au hasard dans la BdG, révèlent une relation positive significative entre les variables indépendantes (produit, prix, lieu et promotion) et la variable dépendante (intentions de déconsommation). De plus, la réglementation amplifie la relation entre les intentions de déconsommation et toutes les variables de la combinaison démarketing (mix demarketing) ${ }^{2}$, à l'exception du produit. Ces résultats sont plus élevés pour les femmes et les jeunes consommateurs, les personnes mariées, les ménages dont le chef de famille est une femme ainsi que les personnes à faible niveau d'instruction et de revenu.

\begin{abstract}
$\boldsymbol{A B S T R A C T}$. Increasing demand for electricity brings up challenges in some areas of the world. Especially in the Gaza Strip (GS) in Palestine, electricity supply shortages are exacerbated by high population growth and by the 10-year Israeli siege that has prevented the upgrading of the only power plant in the GS. Currently, reducing electricity consumption is the only realistic solution to the power shortage. Considering that households account for a significant portion of electricity consumption, this paper investigates if their consumption intentions can be reduced using demarketing strategies, that is to say dissuasion. Furthermore, the paper also tests the effectiveness of regulation in influencing residential deconsumption intentions. An empirical study was conducted using a face-to-face survey to collect primary data to test the bypotheses. The questionnaire was distributed to 384 randomly selected residents in the GS. The findings revealed a significant positive relationship between the independent variables (product, price, place, and promotion) and the dependent variable (deconsumption intentions). Regulation beliefs amplify the relationship between deconsumption intentions and all demarketing mix variables, except for product. These results are higher for women and young consumers, married people, households with a female as head of family, and people with lower education and income.
\end{abstract}

\section{Introduction}

La consommation d'électricité des ménages représente le tiers de la consommation totale d'électricité dans le monde (Nilsson et collab., 2017) et elle n'a cessé d'augmenter en raison de la croissance de la population et de l'utilisation des appareils électroménagers (Tesfamichael et collab., 2020). Ainsi, les consommateurs domestiques constituent une partie prenante importante, et les objectifs de

${ }^{\text {a }}$ Professeur de marketing, Collège universitaire de sciences appliquées, Gaza, Palestine 
durabilité liés à la consommation d'électricité ne peuvent être atteints sans leur participation. Leur contribution est donc essentielle pour le développement de politiques électriques efficaces (Heylen et collab., 2020) sur les économies d'énergie, qui sont cruciales pour réduire la demande globale (Gutiérrez-Pedrero et collab., 2018) et les effets du réchauffement climatique (Doggart et collab., 2020; Afful-Dadzie et collab., 2020). Il est alors nécessaire d'obtenir la contribution des consommateurs pour une consommation d'électricité durable dans un territoire donné.

La demande d'électricité dépassant l'offre n'a pas été étudiée efficacement (Craig et Feng, 2017). Les recherches existantes ne se concentrent pas sur les endroits où l'approvisionnement est sévèrement restreint, par exemple les Territoires palestiniens et la bande de Gaza (BdG). En effet, l'approvisionnement de la centrale électrique a été insuffisant au cours de la dernière décennie, avec des coupures de courant allant jusqu'à 16 heures par jour (Ismail et collab., 2013). Depuis janvier 2020, la disponibilité moyenne de l'approvisionnement est de 11 heures par jour pour un ménage de la BdG (OCHA, 2020). Les réseaux électriques n’ont pas pu être améliorés pour les raisons suivantes (JICA, 2017) :

1. La capacité requise dans la centrale électrique pour desservir les charges est insuffisante, avec un déficit moyen d'environ 75 à $85 \%$ en juillet 2017;

2. Les sources d'énergie sont instables; et

3. L'isolement de la BdG alors qu'Israël l'entoure crée des défis à long terme pour obtenir les matériaux et les fournitures nécessaires.

La perspective de moderniser la seule centrale électrique de la $\mathrm{BdG}$ ou de se connecter à une alimentation électrique plus externe semble peu réaliste et reste soumise à des manœuvres politiques incertaines. De plus, l'adoption des énergies renouvelables comme source alternative d'approvisionnement en électricité est limitée par le siège des autorités israéliennes sur la centrale électrique.

La mise en œuvre de stratégies de conservation de l'énergie est le moyen le plus réaliste et pratique de s'attaquer au dilemme de la demande d'électricité des Territoires palestiniens (Ismail et collab., 2013). Nous proposons que cela doive être fait en tenant compte de la contribution des consommateurs. Le démarketing utilise des outils de marketing, à savoir le produit, le prix, la promotion et le lieu (distribution) $^{3}$, pour dissuader les clients de consommer ou d'acheter certains produits, soit parce que ces produits sont nocifs, soit simplement parce que la demande des consommateurs dépasse l'offre (Dua et collab., 2007). Les stratégies de démarketing peuvent jouer un rôle essentiel dans la rationalisation de la consommation en réduisant l'offre de produits, en augmentant les prix, en lançant des campagnes de sensibilisation et en orientant les préférences des consommateurs vers des produits alternatifs (Yousif, 2014). Compte tenu des limites importantes de l'approvisionnement en électricité, comment freiner efficacement la demande? En s'appuyant sur le cadre théorique du démarketing, notre étude s'attache à répondre à cette question.

Plusieurs lacunes dans la littérature sont abordées dans la présente étude. Tout d'abord, les recherches antérieures n'ont pas étudié en profondeur la réponse des consommateurs aux stratégies de démarketing de l'électricité, en particulier celles mises en œuvre par des organisations privées plutôt que par le gouvernement (Wall, 2005, 2007; Gerstner et collab., 1993). Les données empiriques sur l'utilisation réelle du démarketing sont limitées, et les facteurs potentiels qui influencent quand et comment le démarketing est appliqué manquent également d'attention de la part de la recherche (Kern, 2006).

Deuxièmement, la réaction des consommateurs à la réglementation et leur interaction avec les stratégies de démarketing pour influencer la déconsommation d'électricité n'ont pas été étudiées. Prises ensemble, ces stratégies de démarketing peuvent néanmoins avoir des effets synergiques importants pour favoriser la déconsommation d'électricité. Cependant, Maloney (2014) a étudié l'impact de la réglementation dans le contexte de la durabilité environnementale et s'est concentrée sur la production d'électricité, plutôt que sur la consommation.

Troisièmement, la nature spécifique du problème d'offre et de demande d'électricité auquel fait face la BdG n'a pas été prise en compte dans ses dimensions politiques et économiques.

Notre étude s'efforce de combler ces lacunes de la recherche. Elle apporte trois contributions. Dans un premier temps, nous développons le modèle de Kotler et Levy (1971) pour décommercialiser la consommation d'électricité en ajoutant la régulation 
comme variable modératrice. Le modèle offre une bonne compréhension de la relation entre les facteurs de décommercialisation de l'électricité et la réponse des consommateurs en matière d'intentions de déconsommation. De plus, la présente étude considère le démarketing du point de vue des organisations privées (plutôt que du gouvernement).

Deuxièmement, nous considérons le rôle des croyances relatives au rôle du cadre réglementaire (croyances relatives à la législation) comme modérateur entre les facteurs de démarketing et les intentions de déconsommation des consommateurs. Plus précisément, nous identifions les éléments de la combinaison démarketing qui exercent l'impact le plus décisif sur les intentions de déconsommation, puis montrons comment ces éléments interagissent avec les croyances relatives à la législation. Ainsi, nous évaluons les synergies potentielles entre le démarketing mis en œuvre par le secteur privé du gouvernement et par la législation.

Troisièmement, la présente étude se concentre sur la nature du problème d'offre et de demande d'électricité dans la centrale électrique. La région de la BdG a un besoin urgent de recherches supplémentaires en raison de défis importants résultant de son incapacité à résoudre une telle situation en développant les installations de fourniture d'électricité ou en introduisant des énergies renouvelables à grande échelle (en raison de restrictions politiques). Ainsi, pour la BdG, la mise en œuvre de la stratégie de démarketing constitue peut-être la seule solution viable pour rationaliser la consommation. Mise en œuvre efficacement, elle pourrait atténuer l'impact drastique de la pénurie d'électricité sur l'ensemble de la société.

Le reste de l'article est structuré comme suit. Le cadre conceptuel et les hypothèses sont décrits dans la section 2. La méthodologie est discutée dans la section 3, y compris l'échantillon de l'étude, les mesures et la collecte de données. Les résultats de l'étude sont présentés dans la section 4, suivis d'une discussion dans la section 5, y compris les implications théoriques, managériales, pratiques et politiques de l'étude ainsi que la portée de la recherche future. Dans la section 6, des remarques de conclusion, de limites et de pistes de recherche sont fournies.

\section{Cadre conceptuel et développement des hypothèses}

Dans cette section, le contexte théorique de la stratégie de démarketing est présenté. Chaque élément de démarketing est discuté, et les hypothèses sont formulées en relation avec la recherche empirique et la théorie existante. Une prédisposition pertinente du consommateur est également discutée, puis des hypothèses spécifiques en sont dérivées.

\subsection{La théorie du démarketing}

Selon Kotler (2017), le démarketing est synonyme de réduction de la demande. Il est défini comme «l'aspect du marketing qui consiste à décourager la clientèle en général ou une certaine catégorie de clients en particulier, sur une base temporaire ou permanente» (Kotler et Levy, 1971, p. 75, trad. libre). Cette définition est largement acceptée par les spécialistes du marketing et par les praticiens (p. ex., Bradley et Blythe, 2013). Nous l'adopterons dans la présente étude.

Le démarketing est mis en œuvre par les organisations et/ou par les gouvernements en modulant les éléments de la combinaison marketing (c.-à-d. le produit, le prix, le lieu et la promotion). Quatre situations appellent un démarketing (Kotler, 2017) :

1. Gérer une pénurie existante;

2. Éviter les pénuries potentielles;

3. Minimiser les dommages aux individus;

4. Minimiser les dommages causés à la nature ou aux ressources uniques.

Alors que nous vivons dans un monde où les ressources terrestres et les produits diminuent et peuvent sérieusement affecter notre santé, le démarketing devient la nouvelle norme.

Cependant, il comporte quelques mises en garde (Kotler, 2017). Tout d'abord, la campagne de démarketing peut rendre le produit ou le service plus attrayant (p. ex., interdire un livre ou un film). Deuxièmement, les efforts de démarketing pourraient stimuler l'émergence d'une classe criminelle qui prospérera pendant la rareté induite (p. ex., le cas pendant l'ère de la prohibition aux États-Unis lorsque l'alcool a été interdit). Troisièmement, certains défenseurs des droits de la personne, des libéraux et des libertariens pourraient discuter de l'ingérence du gouvernement 
dans ce qu'ils considèrent comme les droits fondamentaux des citoyens et citoyennes. Or, aucune de ces mises en garde ne s'applique à la présente étude.

De plus, les stratégies de démarketing visant à rationaliser la consommation d'électricité dans la bande de Gaza sont actuellement la seule option disponible pour faire face à la pénurie d'approvisionnement en électricité. Kotler (2017) déclare que le démarketing fonctionne mieux lorsqu'il existe un consensus citoyen élevé selon lequel la consommation de certains biens ou services devrait être réduite. Par l'obtention de l'avis des consommateurs sur les actions de démarketing potentielles, cette étude tente précisément d'y parvenir.

La recherche empirique sur le démarketing de l'électricité s'est développée au cours des dernières décennies, en particulier avec l'émergence du marketing social, du marketing éthique et de la durabilité dans le marketing (p. ex., Grinstein et Nisan, 2009; Ramirez et collab., 2017; Al-Samydai et Yousif, 2018; Salem et collab., 2021). Alors que le démarketing de l'électricité est d'une importance cruciale pour les Palestiniens et Palestiniennes ainsi que la population des pays arabes voisins, il semble que la pénurie d'eau soit une préoccupation plus grave en Israël (Grinstein et Nisan, 2009).

Dans ce contexte, ces deux auteurs ont exploré comment différents groupes au sein du pays multiethnique d'Israël réagissent à la campagne de démarketing du gouvernement visant à réduire la consommation d'eau. De leur côté, Ramirez et ses collègues (2017) ont étudié l'efficacité du démarketing proenvironnemental sur le comportement de consommation d'électricité des consommateurs et ont trouvé des effets significatifs. Al-Samydai et Yousif (2018) ont évalué l'impact d'une stratégie générale de démarketing sur la demande d'électricité sur un échantillon jordanien. La stratégie de démarketing utilisée était composée de cinq éléments : la publicité, la perception, le prix, l'approvisionnement en énergie solaire et le groupe de référence, ce qui a effectivement réduit la demande d'électricité. Enfin, Salem et ses collègues (2021) ont étudié les stratégies de démarketing de l'électricité dans la $\mathrm{BdG}$ sous diverses variables psychographiques, y compris la sensibilisation, la motivation et les attitudes des Palestiniens et Palestiniennes envers la compagnie d'approvisionnement en électricité.
Cependant, aucune de ces études n'a pris en compte les stratégies de démarketing en relation avec la réglementation gouvernementale. Contrairement aux recherches antérieures, et en tant que tentative de compléter l'état de l'art, cette étude propose la réglementation comme un modérateur critique avec le potentiel de renforcer les efforts de gestion de démarketing pour la déconsommation d'électricité.

\subsection{La relation entre la combinaison démarketing et la réponse des consommateurs}

Cette étude se concentre sur les stratégies de démarketing des organisations, et non du gouvemement. Nous stipulons des effets directs des éléments de démarketing du produit (H1), du prix (H2), du lieu (H3) et de la promotion (H4) sur les intentions de déconsommation résidentielle de l'électricité.

\section{Leproduit}

En démarketing, le produit correspond à des efforts de marketing pour réduire son volume de vente. Kern (2006) affirme que les entreprises essaient de limiter les lignes d'approvisionnement ainsi que de minimiser les activités de service et de production, ce qui encourage l'achat du produit. De plus, il est bien établi que la clientèle satisfaite a un impact positif sur la rentabilité, car elle préfère acheter plus d'exemplaires du produit et s'engager dans des rachats à long terme (Khadka et Maharjan, 2017). En revanche, la clientèle insatisfaite réduit les revenus des entreprises en raison de la baisse de ses achats et de sa consommation de produits (Khadka et Maharjan, 2017). De plus, l'insatisfaction contribue souvent aux plaintes (Donoghue et de Klerk, 2006) et au bouche-à-oreille défavorable (Richins, 1983). Ainsi, si la satisfaction augmente la consommation et que l'insatisfaction la diminue, un client insatisfait aurait plus de chances de formuler des intentions de déconsommation.

Par ailleurs, faciliter et encourager l'utilisation de produits alternatifs est une stratégie de démarketing critique (Silagy et Stead, 2001; Lancaster et collab., 2000). L'effet positif de la modulation des produits sur les intentions de déconsommation d'électricité a été établi empiriquement (Salem et collab., 2021). Sur la base de ce qui précède, nous émettons l'hypothèse :

H1 : La modulation des produits affecte positivement les intentions de déconsommation résidentielle d'électricité. 
Leprix

La détermination des prix peut présenter une solution simple mais efficace à la déconsommation d'électricité. La stratégie de prix de démarketing se concentre sur la diminution de la demande actuelle en augmentant les prix (Salem et collab., 2018). Comme Maxwell (2002) l'a noté, la tarification a un impact sur les attitudes et le comportement de la clientèle. Si celle-ci considère le prix comme déraisonnablement élevé, elle aura probablement une attitude négative envers le produit, ce qui mènera à une faible intention d'achat (Xia et collab., 2004). D'un point de vue strictement économique, l'augmentation des prix diminuera la demande (p. ex., Monfared et Ghasemi, 2019).

Pourtant, la détermination des prix dans le démarketing constitue un des processus les plus difficiles pour les décideurs (Niroomand et Jenkins, 2020). L'objectif de la politique de prix de démarketing est d'augmenter les prix pour réduire, mais pas d'éliminer la demande de produits.

Dans certains pays, les fournisseurs de services électriques continuent d'augmenter les prix pour inciter la clientèle à rationaliser sa consommation d'électricité (Sovacool, 2017). Par exemple, dans la BdG, Salem et ses collègues (2021) ont établi que l'évolution des prix en fonction du niveau de consommation stimule les intentions de déconsommation résidentielle d'électricité. Ainsi, nous posons l'hypothèse suivante :

H2: La modulation des prix affecte positivement les intentions de déconsommation résidentielle d'électricité.

\section{Le lien}

Selon certaines recherches, le lieu/l'emplacement constitue un des aspects critiques du démarketing. Il peut être modulé en tant que lieu de consommation ou lieu d'achat, et les deux éléments peuvent imposer un effet de démarketing (Pechmann et Knight, 2002). Selon Ajzen (1991), limiter les zones pour adopter un comportement peut constituer un obstacle à la promotion du comportement et avoir un impact profond sur les intentions. Ainsi, les restrictions de consommation sont un frein essentiel à la consommation et devraient avoir un impact direct sur les intentions de consommation (Shiu et collab., 2009).

De plus, les restrictions à l'achat auraient un résultat similaire; elles limiteraient l'influence attribuable au coût d'opportunité (p. ex., Berry et collab.,
2002). En démarketing, cela implique que consacrer plus de temps et d'efforts à la recherche d'un produit minimiserait le temps consacré à d'autres tâches telles que les loisirs, les tâches ménagères ou l'entretien personnel (Shiu et collab., 2009). La modulation de lieu freine les intentions de déconsommation dans le cas particulier de la consommation électrique résidentielle (Salem et collab., 2021). Ainsi, nous proposons l'hypothèse suivante :

H3 : La modulation de lieu affecte positivement les intentions de déconsommation résidentielle d'électricité.

\section{La promotion}

La stratégie de démarketing comprend la promotion pour inciter la clientèle à acheter des produits non utilisés et des pratiques antipromotionnelles afin de rationaliser la consommation (Yousif, 2014). S'appuyant sur Beeton et Pinge (2003) et Beeton et Benfield (2002), Kern (2006) souligne que, à titre d'un des principaux objectifs du marketing environnemental, la promotion se concentre plutôt sur l'information donnée au public concernant la valeur du comportement environnemental ainsi que la nécessité de protéger l'environnement et les ressources naturelles.

La recherche démontre l'importance en démarketing de la promotion auprès des consommateurs (Page et Colby, 2003; Siegel et Biener, 2000) en raison de son impact négatif direct sur les intentions (Andrews et collab., 2004). En effet, une perception plus élevée des efforts de promotion et de communication pour déconsommer conduira les consommateurs à penser à diminuer leur consommation d'électricité (Salem et collab., 2021). Nous anticipons donc l'hypothèse suivante :

\section{H4 : La modulation de la promotion affecte positivement} les intentions de déconsommation résidentielle d'électricité.

\subsection{L'effet modérateur des croyances relatives à la législation}

La législation promue par le gouvernement est susceptible non seulement d'influencer directement la déconsommation d'électricité des consommateurs, mais également d'amplifier l'effet des mesures de démarketing. Elle est définie au sens large comme «l'activité intentionnelle consistant à tenter de contrôler, d'ordonner ou d'influencer le comportement d'autrui» (Black, 2002, p. 19, trad. libre). 
Quant au terme réglementation, il couvre un concept plus large que celui de loi (Ayres et Braithwaite, 1992) et est donc adopté dans cette étude. En plus des lois formellement établies, par exemple les statuts - réglementations primaire et déléguée - et les lois communes, la législation inclut des incitations économiques et des cadres basés sur le marché (Vandenbergh, 2004).

Supposons que les croyances relatives à la législation concernant un produit ou un service spécifique devraient accroître la sensibilité des consommateurs envers ce produit et, par conséquent, rendre ses caractéristiques (le produit lui-même, le prix, le lieu et la distribution) plus importantes pour le consommateur. Par conséquent :

H5 : Les croyances relatives à la législation amplifient l'effet (a) du produit, (b) du prix, (c) du lieu et (d) de la promotion sur les intentions de déconsommation résidentielle d'électricité.

Le cadre conceptuel de l'étude est illustré dans la figure 1. Ce modèle relie la combinaison démarketing d'une organisation aux intentions de déconsommation d'électricité des consommateurs. Il montre également l'effet de modération exercé par la régulation sur chacun des chemins hypothétiques des variables de la combinaison démarketing jusqu'aux intentions de déconsommation.

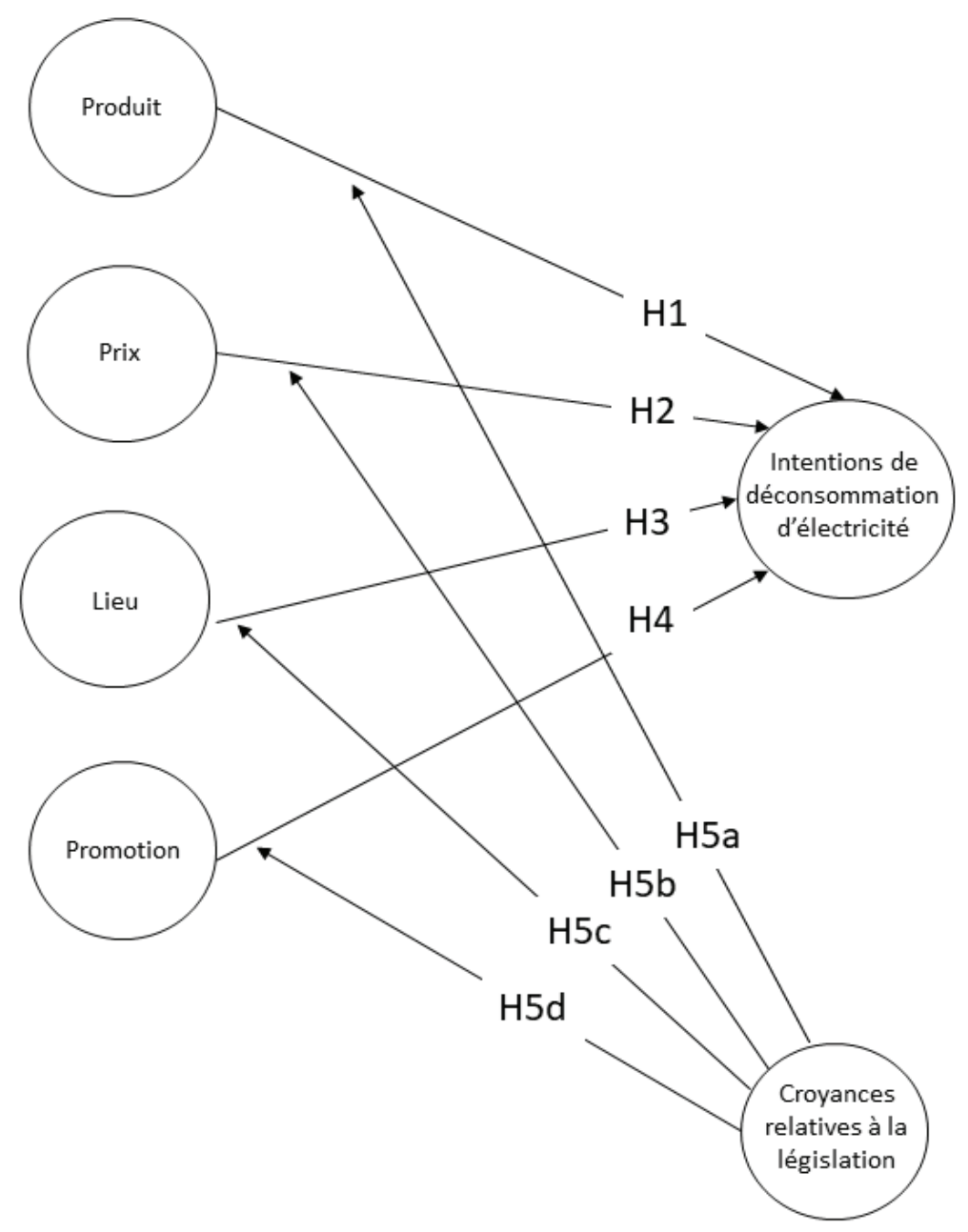

Figure 1-Cadre conceptuel de l'étude 


\section{Méthodologie}

\subsection{Le contexte territorial de l'étude}

L'étude a été menée dans la BdG, en Palestine, qui se distingue par des caractéristiques particulières qui la rendent unique. Contrairement à la Cisjordanie, qui est un territoire palestinien occupé (Alvi et collab., 2019), la BdG est une enclave inoccupée qui résiste à l'occupation depuis maintenant des décennies.

Par conséquent, contrairement au contexte extrême de colonisation territoriale progressive au sein de la Cisjordanie (Alvi et collab., 2019), la BdG fait face à des restrictions territoriales strictes imposées par le gouvernement israélien, ce qui limite sévèrement l'approvisionnement en électricité.

La demande énergétique quotidienne de la centrale électrique était d'environ 450 mégawatts (MW) en 2017. Pourtant, l'offre quotidienne d'énergie n'a pas dépassé les 210 MW depuis 2006. Si l'offre actuelle de 210 MW n'augmente pas, la pénurie de la demande d'électricité s'aggravera bientôt. Plus précisément, les trois quarts de la demande en 2025 ne seront pas satisfaits (JICA, 2017).

Avant la division des Territoires palestiniens en 2007 en deux entités (à savoir la Cisjordanie, gouvernée par l'Autorité palestinienne, et la BdG, gouvernée par le parti du Hamas), les 210 MW quotidiens disponibles pour la BdG étaient fournis comme suit : $90 \mathrm{MW}$ de la seule centrale électrique de Gaza (qui produit environ $43 \%$ de l'approvisionnement en électricité) et 120 MW de lignes électriques israéliennes externes, financées par l'Autorité palestinienne (UNSCO, 2017). Plusieurs donateurs, dont la Banque mondiale, la Banque islamique de développement, le Bureau de la coordination des affaires humanitaires du Programme des Nations unies pour le développement (BCAH-PNUD), le Qatar et la Turquie, ont soutenu l'achat de matériel de distribution et de transformateurs nécessaires pour la reconstruction du réseau de distribution endommagé.

Néanmoins, la situation de l'alimentation dans la centrale électrique reste gravement détériorée (JICA, 2017). Cette situation est en partie due à l'Ordonnance de contrôle des exportations de défense (Ministry of Defense, 2007), qui restreint l'expédition de certains produits dans la $\mathrm{BdG}$, notamment le ciment, les agrégats, les éléments en acier et les matériaux de construction, les fils d'acier ou les véhicules autres que ceux à usage personnel. Ainsi, la livraison des biens et matériels essentiels dans la BdG pour la mise en œuvre du projet est limitée.

Essentiellement, les restrictions politiques imposées à la centrale électrique limitent la possibilité de faire face à la grave pénurie d'électricité en agrandissant les centrales de production d'électricité ou en introduisant des systèmes d'énergies renouvelables à grande échelle. Cela définit la BdG comme un contexte idéal pour mettre en œuvre des stratégies de démarketing par les fournisseurs privés, en coordination avec la réglementation gouvernementale, pour réduire la consommation d'électricité.

\subsection{Les instruments de mesure}

Un questionnaire a été conçu pour évaluer les réponses des consommateurs aux efforts de démarketing et à la législation concernant la consommation d'électricité. La principale variable dépendante de cette étude est l'intention de déconsommer de l'électricité. Le choix des intentions est basé sur des preuves convaincantes et solides dans la littérature, qui ont démontré que les intentions comportementales sont de bons prédicteurs du comportement réel, comme Ajzen (1991) l'a proposé initialement.

Les variables indépendantes sont constituées des quatre éléments de la combinaison démarketing et sont mesurées en fonction de la façon dont les consommateurs les perçoivent. Mesurer la perception a du sens, car elle représente la réalité telle qu'elle est perçue par les consommateurs.

1. La variable produit a été mesurée avec une échelle à quatre items adaptée de Salem et ses collègues (2018) et de Seeletse (2016);

2. La variable prix a été évaluée selon une échelle à six items adaptée d'Al-Samydai et Yousif (2018), de Salem (2013) et de Kim et Shin (2016);

3. La variable lieu a été évaluée par une échelle à quatre items adaptée de Medway et ses collègues (2011);

4. Une échelle à trois items a été adaptée de Salem et ses collègues (2018) et de Shiu et ses collègues (2009) pour mesurer la variable promotion;

5. La variable modératrice des croyances relatives à la législation a été mesurée à l'aide d'une échelle à trois items adaptée de Maloney (2014) et de Beeton et Benfield (2002) sur les croyances des consommateurs à l'égard de la législation. 
Toutes les mesures ont été prises à l'aide d'une échelle de Likert à sept points allant de 1 («pas du tout d'accord») à 7 («tout à fait d'accord»). En outre, diverses informations démographiques ont été évaluées, dont l'âge, le genre, le niveau d'éducation, l'état matrimonial, le revenu mensuel et l'indépendance financière. Enfin, des informations spécifiques au contexte de la recherche ont été obtenues, y compris l'état des dettes sur les factures d'électricité et le taux quotidien (heure/jour) de coupure d'électricité des ménages interrogés.

\subsection{La collecte de données et l'échantillonnage}

Les hypothèses de recherche ont été testées selon une méthodologie de recherche descriptive transversale basée sur une enquête. Les données ont été collectées à l'aide d'un questionnaire lors de visites de terrain en face à face pour assurer un taux de réponse élevé (Saunders et collab., 2016). Le questionnaire a été traduit en arabe, qui est la langue locale, par un traducteur professionnel, puis revu par deux locuteurs natifs arabes. Enfin, un test pilote a été effectué pour la traduction et l'exactitude du contenu.

Une équipe de 20 personnes ayant une expérience en marketing a effectué la passation du questionnaire.
Les membres de l'équipe ont été divisés en cinq groupes. Chaque groupe était composé de trois enquêteurs et d'un superviseur. Un groupe a été affecté à chaque gouvernorat de la BdG. Les membres de l'équipe ont participé à un programme de formation de deux jours sur les procédures de collecte de données. Les superviseurs de groupe étaient chargés d'assurer la qualité du processus de collecte de données, de le superviser strictement et de s'assurer que l'étude n'est pas affectée par les biais de l'enquêteur.

La population de l'étude comprenait l'ensemble des 1899291 habitants (PCBS, 2018) de la BdG. Un échantillon aléatoire stratifié de 384 habitants a été sélectionné dans la population en fonction du pourcentage d'habitants dans chacun des cinq gouvernorats de la BdG (voir Tableau 1). L'échantillon a été calculé selon un intervalle de confiance à $95 \%$ en utilisant la formule de détermination de l'échantillon, ce qui donnera au chercheur une marge d'erreur de $5 \%$ (Saunders et collab., 2016). Les entretiens pour la collecte de données ont été menés sur une période d'un mois (du 5 mars 2020 au 6 avril 2020). Sur les 384 questionnaires distribués, 359 ont été retournés complètement remplis et exacts. Les 359 répondants sont suffisamment représentatifs de la démographie réelle de la BdG (voir Tableau 2).

\begin{tabular}{|c|c|c|c|c|}
\hline Gouvernorat & Genre & Population* & $\%$ & $\begin{array}{c}\text { Taille de } \\
\text { l'échantillon }\end{array}$ \\
\hline \multirow{3}{*}{ Gaza Nord } & $\mathrm{H}$ & 187763 & \multirow[t]{3}{*}{19,4} & \multirow[t]{3}{*}{75} \\
\hline & $\mathrm{F}$ & 181215 & & \\
\hline & Total & 368978 & & \\
\hline \multirow{3}{*}{ Gaza } & $\mathrm{H}$ & 331985 & \multirow[t]{3}{*}{34,4} & \multirow[t]{3}{*}{132} \\
\hline & $\mathrm{F}$ & 320612 & & \\
\hline & Total & 652597 & & \\
\hline \multirow{3}{*}{ Deir al-Balah } & $\mathrm{H}$ & 137340 & \multirow[t]{3}{*}{14,4} & \multirow[t]{3}{*}{55} \\
\hline & $\mathrm{F}$ & 135860 & & \\
\hline & Total & 273200 & & \\
\hline \multirow{3}{*}{ Khan Yunis } & $\mathrm{H}$ & 187964 & \multirow[t]{3}{*}{19,5} & \multirow[t]{3}{*}{75} \\
\hline & $\mathrm{F}$ & 182674 & & \\
\hline & Total & 370638 & & \\
\hline \multirow{3}{*}{ Rafah } & $\mathrm{H}$ & 117851 & \multirow[t]{3}{*}{12,3} & \multirow[t]{3}{*}{47} \\
\hline & $\mathrm{F}$ & 116027 & & \\
\hline & Total & 233878 & & \\
\hline \multicolumn{2}{|l|}{ Total } & 1899291 & 100 & 384 \\
\hline
\end{tabular}

*Données tirées de PCBS (2018)

Tableau 1- Distribution de l'échantillon par gouvernorat 


\begin{tabular}{|c|c|c|c|}
\hline Variables & Catégories & Fréquence & $\%$ \\
\hline \multirow{6}{*}{ Âge } & 20 ans ou - & 5 & 1,5 \\
\hline & $21-30$ ans & 139 & 42,5 \\
\hline & $31-40$ ans & 115 & 35,1 \\
\hline & $41-50$ ans & 31 & 9,5 \\
\hline & $51-60$ ans & 25 & 7,7 \\
\hline & 61 ans et + & 12 & 3,7 \\
\hline \multirow{2}{*}{ Genre } & Homme & 167 & 51,1 \\
\hline & Femme & 160 & 48,9 \\
\hline \multirow{5}{*}{ Niveau d'éducation } & Secondaire ou - & 53 & 16,2 \\
\hline & $\begin{array}{l}\text { Diplôme (inférieur au } \\
\text { baccalauréat/licence) }\end{array}$ & 98 & 30,0 \\
\hline & Baccalauréat (licence) & 163 & 49,9 \\
\hline & Maîtrise (master) & 8 & 2,5 \\
\hline & Ph. D./doctorat & 5 & 1,4 \\
\hline \multirow{4}{*}{ État matrimonial } & Célibataire & 99 & 30,3 \\
\hline & Marié(e) & 198 & 60,5 \\
\hline & Veuf(ve) & 17 & 5,2 \\
\hline & Divorcé(e) & 13 & 4,0 \\
\hline \multirow{5}{*}{ Revenu mensuel* } & $500 \$$ ou - & 194 & 59,3 \\
\hline & $501 \$-1000 \$$ & 108 & 33,0 \\
\hline & $1001 \$-1500 \$$ & 17 & 5,2 \\
\hline & $1501 \$-2000 \$$ & 8 & 2,5 \\
\hline & $2001 \$$ et + & 0 & 0 \\
\hline \multirow{2}{*}{ Indépendance financière } & Oui & 228 & 69,7 \\
\hline & Non & 99 & 30,3 \\
\hline \multirow{3}{*}{ Paiement de votre électricité } & Oui & 143 & 43,7 \\
\hline & Non & 97 & 29,7 \\
\hline & Parfois & 87 & 26,6 \\
\hline \multirow{3}{*}{$\begin{array}{l}\text { Nbre d'heures/jour durant } \\
\text { lesquelles l'électricité est coupée }\end{array}$} & Moins de $8 \mathrm{~h}$ & 140 & 42,8 \\
\hline & $8-16 \mathrm{~h}$ & 149 & 45,6 \\
\hline & Plus de $16 \mathrm{~h}$ & 38 & 11,6 \\
\hline \multirow{3}{*}{ Perception des prix de l'électricité } & Élevée & 249 & 76,2 \\
\hline & Moyenne & 78 & 23,8 \\
\hline & Faible & 0 & 0 \\
\hline
\end{tabular}

* Les montants ont été convertis en dollars canadiens pour plus de clarté.

Tableau 2- Profil des répondants $(n=359)$

\subsection{L’analyse des données}

Les données recueillies ont été analysées sur la base de la modélisation PLS-SEM à l'aide du logiciel SmartPLS 3.0 (Ringle et collab., 2015). Une approche d'analyse en deux étapes (Chin, 1998) a été utilisée pour évaluer les modèles d'équations structurelles.

Dans un premier temps, des tests de validité et de fiabilité ont été menés sur les items de mesure (Ringle et collab., 2015). Des tests ont été effectués pour vérifier la validité convergente et discriminante du modèle de mesure. De plus, des mesures de cohérence interne et de fiabilité de construction ont été prises en compte. Pour évaluer la validité discriminante, le critère de Fornell-Larcker a été utilisé. Il consiste à comparer la racine carrée de la variance moyenne extraite (VME) et la variance partagée (c.-à-d. la corrélation) entre les construits latents apparentés.

Deuxièmement, des tests ont été effectués sur les modèles d'équations structurelles pour vérifier les hypothèses. Les relations entre les hypothèses du 
modèle de recherche ont été prédites sur la base du coefficient de détermination $\mathrm{R}^{2}$. Pour évaluer tous les coefficients de chemins hypothétiques, la technique de bootstrapping a été utilisée sur 5000 rééchantillons.

\section{Résultats}

\subsection{Le modèle de mesure}

Tous les indicateurs des construits ont été inclus dans un modèle global d'analyse factorielle confirmatoire (AFC) pour évaluer les propriétés psychométriques des mesures. Le logiciel SmartPLS 3.0 a été utilisé pour évaluer le modèle de mesure (voir Tableau 3). Ce logiciel est basé sur la méthode de modélisation du chemin PLS, qui consiste en une séquence de régressions dans les vecteurs de poids (Henseler et collab., 2009).

L'AFC indique que l'échelle correspond aux données de manière appropriée. Tous les éléments sauf 8 sur 41 étaient au-dessus de la charge factorielle recommandée de 0,70 (Nunnally, 1978). Plus important encore, tous étaient significatifs selon un intervalle de confiance de $99 \%$, soit bien au-delà du seuil initial de $95 \%$, ce qui suggère une validité convergente élevée. La variance expliquée par les facteurs dépassait le critère recommandé de $50 \%$ dans chaque cas (Henseler et collab., 2009). De tels résultats garantissent conjointement que les construits présentent une validité convergente.

Les coefficients de fiabilité allaient de 0,775 à 0,931, et les valeurs de l'alpha de Cronbach $(\alpha)$ étaient toutes supérieures ou légèrement proches au seuil de 0,60 spécifié par Nunnally et Bernstein (1994). Selon les résultats, les constructions présentent des propriétés de fiabilité souhaitables. La validité discriminante a été évaluée par le critère de Fornell et Larcker (1981), qui stipule que la VME de chaque construit latent doit être supérieure à la corrélation la plus élevée entre tous les autres construits. Comme le montre la matrice de corrélations présentée au tableau 4, aucune VME n'était inférieure à la corrélation la plus élevée entre tout autre construit, ce qui confirme la validité discriminante. 


\begin{tabular}{|c|c|c|c|c|c|}
\hline Construit & $\begin{array}{l}\text { Items } \\
\end{array}$ & CFS & FC & $\alpha$ & VME \\
\hline \multirow[t]{4}{*}{ Produit } & Je ne suis pas satisfait(e) du service de fourniture d'électricité actuel. & 0,855 & \multirow{4}{*}{0,922} & \multirow{4}{*}{0,890} & \multirow{4}{*}{0,748} \\
\hline & $\begin{array}{l}\text { La fourniture d'électricité fournie est inférieure à ma demande } \\
\text { quotidienne. }\end{array}$ & 0,819 & & & \\
\hline & $\begin{array}{l}\text { Je réduirai ma consommation d'électricité si l’approvisionnement } \\
\text { quotidien en électricité est plus constant. }\end{array}$ & 0,912 & & & \\
\hline & $\begin{array}{l}\text { Je réduirai ma consommation d'électricité si le calendrier des périodes } \\
\text { de fourniture d'électricité est sans ambiguité. }\end{array}$ & 0,870 & & & \\
\hline \multirow[t]{6}{*}{ Prix } & De mon point de vue, le coût unitaire de l'électricité est élevé. & 0,858 & \multirow{6}{*}{0,874} & \multirow{6}{*}{0,824} & \multirow{6}{*}{0,541} \\
\hline & $\begin{array}{l}\text { De mon point de vue, la facture d'électricité est différente de ma } \\
\text { consommation d'électricité. }\end{array}$ & 0,768 & & & \\
\hline & $\begin{array}{l}\text { De mon point de vue, la facture d'électricité est différente des } \\
\text { périodes de fourniture. }\end{array}$ & 0,726 & & & \\
\hline & $\begin{array}{l}\text { Je réduirai ma consommation d'électricité si les prix sont basés sur } \\
\text { l'utilisation (commerciale, industrielle, agricole, résidentielle). }\end{array}$ & 0,719 & & & \\
\hline & $\begin{array}{l}\text { Je réduirai ma consommation d'électricité si des prix plus élevés } \\
\text { s'appliquent pour des niveaux de consommation plus élevés. }\end{array}$ & 0,744 & & & \\
\hline & $\begin{array}{l}\text { Offrir des incitations à des prix plus bas aux utilisateurs de faible } \\
\text { niveau de consommation peut contribuer à résoudre le problème de } \\
\text { la pénurie d'électricité. }\end{array}$ & 0,828 & & & \\
\hline \multirow[t]{4}{*}{ Lieu } & $\begin{array}{l}\text { L'augmentation des sources d'électricité, y compris l'option } \\
\text { d'importation, pourrait contribuer à résoudre le problème de pénurie } \\
\text { d'électricité. }\end{array}$ & 0,737 & \multirow{4}{*}{0,784} & \multirow{4}{*}{0,715} & \multirow{4}{*}{0,508} \\
\hline & $\begin{array}{l}\text { Améliorer l'efficacité des réseaux électriques pour réduire le } \\
\text { pourcentage d'électricité gaspillée est crucial pour résoudre le problème. }\end{array}$ & 0,793 & & & \\
\hline & $\begin{array}{l}\text { Une répartition équitable de l'électricité en fonction du nombre } \\
\text { d'habitants et de leur répartition géographique peut aider à résoudre } \\
\text { le problème. }\end{array}$ & 0,787 & & & \\
\hline & $\begin{array}{l}\text { Empêcher les citoyens de se connecter illégalement aux lignes } \\
\text { électriques contribue à résoudre le problème. }\end{array}$ & 0,801 & & & \\
\hline \multirow[t]{3}{*}{ Promotion } & $\begin{array}{l}\text { Les campagnes promotionnelles devraient être utilisées efficacement } \\
\text { pour illustrer les avantages économiques et sociaux obtenus grâce à la } \\
\text { rationalisation de la consommation d'électricité. }\end{array}$ & 0,753 & \multirow{3}{*}{0,771} & \multirow{3}{*}{0,715} & \multirow{3}{*}{0,532} \\
\hline & $\begin{array}{l}\text { Des campagnes de promotion et des incitations financières devraient } \\
\text { être mises en œuvre par le gouvernement pour encourager la } \\
\text { rationalisation de la consommation d'électricité. }\end{array}$ & 0,810 & & & \\
\hline & $\begin{array}{l}\text { Les réseaux d'institutions gouvernementales et non } \\
\text { gouvernementales devraient développer des campagnes pour } \\
\text { sensibiliser les consommateurs aux conséquences d'une } \\
\text { consommation d'électricité irrationnelle. }\end{array}$ & 0,741 & & & \\
\hline \multirow{3}{*}{$\begin{array}{l}\text { Croyances } \\
\text { relatives à la } \\
\text { législation }\end{array}$} & $\begin{array}{l}\text { Il est nécessaire de promulguer des lois et des politiques qui exhortent } \\
\text { les ministères à rationaliser la consommation d'électricité. }\end{array}$ & 0,788 & \multirow{3}{*}{0,876} & \multirow{3}{*}{0,791} & \multirow{3}{*}{0,703} \\
\hline & $\begin{array}{l}\text { Je soutiens les lois et la législation qui récompensent ceux qui } \\
\text { rationalisent leur consommation d'électricité. }\end{array}$ & 0,886 & & & \\
\hline & $\begin{array}{l}\text { Les lois et la législation jouent un rôle important pour inciter les } \\
\text { consommateurs à rationaliser leur consommation d'électricité. }\end{array}$ & 0,838 & & & \\
\hline \multirow{9}{*}{$\begin{array}{l}\text { Intentions } \\
\text { de } \\
\text { déconsom- } \\
\text { mation } \\
\text { d'électricité }\end{array}$} & $\begin{array}{l}\text { Je reconnais le rôle efficace joué par la rationalisation de la } \\
\text { consommation d'électricité dans la protection de l'économie. }\end{array}$ & 0,729 & \multirow{9}{*}{0,857} & \multirow{9}{*}{0,820} & \multirow{9}{*}{0,514} \\
\hline & $\begin{array}{l}\text { Les campagnes de sensibilisation visant à rationaliser la } \\
\text { consommation d'électricité sont importantes. }\end{array}$ & 0,700 & & & \\
\hline & $\begin{array}{l}\text { Je pense que les lois et la législation actuelles incitent à la } \\
\text { rationalisation de la consommation d'électricité. }\end{array}$ & 0,712 & & & \\
\hline & $\begin{array}{l}\text { L'augmentation des prix de l'électricité joue un rôle essentiel dans la } \\
\text { rationalisation de la consommation d'électricité. }\end{array}$ & 0,761 & & & \\
\hline & $\begin{array}{l}\text { La nécessité de passer à des sources alternatives (comme l'énergie } \\
\text { solaire) est importante. }\end{array}$ & 0,732 & & & \\
\hline & $\begin{array}{l}\text { Le gouvernement devrait appliquer des lois pour mettre en œuvre des } \\
\text { contrôles visant à rationaliser la consommation d'électricité. }\end{array}$ & 0,783 & & & \\
\hline & $\begin{array}{l}\text { Élaborer des mesures de contrôle efficaces pour réduire le } \\
\text { détournement illégal d'électricité. }\end{array}$ & 0,757 & & & \\
\hline & Je peux réduire la consommation d'électricité. & 0,730 & & & \\
\hline & Je peux réduire le gaspillage d'électricité. & 0,707 & & & \\
\hline
\end{tabular}

Tableau 3 - Estimation de la validité de l'échelle de mesure 


\begin{tabular}{lllllllllll}
\hline & P1 & P2 & P3 & P4 & CRL & D & EM1 & EM2 & EM3 & EM4 \\
\hline P1 & 0,865 & & & & & & & & & \\
P2 & 0,422 & 0,736 & & & & & & & & \\
P3 & 0,375 & 0,609 & 0,701 & & & & & & & \\
P4 & 0,173 & 0,326 & 0,445 & 0,730 & & & & & & \\
CRL & 0,200 & 0,463 & 0,647 & 0,398 & 0,838 & & & & & \\
D & 0,188 & 0,492 & 0,561 & 0,530 & 0,475 & 0,636 & & & & \\
EM1 & 0,521 & 0,250 & 0,254 & 0,187 & 0,161 & 0,203 & 1000 & & & \\
EM2 & 0,268 & 0,484 & 0,515 & 0,305 & 0,374 & 0,384 & 0,509 & 1000 & & \\
EM3 & 0,253 & 0,478 & 0,574 & 0,343 & 0,458 & 0,332 & 0,474 & 0,801 & 1000 & \\
EM4 & 0,209 & 0,317 & 0,385 & 0,270 & 0,304 & 0,182 & 0,352 & 0,594 & 0,647 & 1000 \\
\hline
\end{tabular}

Note. P1 = produit; P2 = prix; P3 = lieu; P4 = promotion; CRL = croyances relatives à la législation (dans la rationalisation de la consommation d'électricité); D = démarketing; EM1 = effet modérateur 1; EM2 = effet modérateur 2; EM3 = effet modérateur 3; EM4 = effet modérateur 4.

* La racine carrée de la VME figure en diagonale dans le tableau. Les valeurs hors diagonale constituent les coefficients de corrélation.

Tableau 4-Validité discriminante des construits (racine carrée de la VME et coefficients de corrélation*)

\subsection{Les modèles d'équations structurelles}

Deux modèles distincts sont estimés. Le premier évalue l'effet principal, c'est-à-dire l'effet direct des éléments de la combinaison démarketing sur les intentions de déconsommation. Le second modèle ajoute l'impact de la variable modératrice sur les relations directes. Les résultats des modèles d'équations structurelles sont présentés dans le tableau 5.

Les indices d'ajustement globaux conviennent systématiquement aux deux modèles. Plus précisément, le coefficient $\mathrm{Q}^{2}$ de Stone-Geisser a été utilisé pour la pertinence prédictive, tandis que le résidu quadratique moyen standardisé (RQMS) a été utilisé pour évaluer l'ajustement du modèle. Le coefficient $\mathrm{Q}^{2}$ établit dans quelle mesure les valeurs observées sont reproduites par le modèle et ses paramètres estimés (Geisser, 1975). Une valeur du $\mathrm{Q}^{2}$ supérieure à 0 indique une pertinence prédictive (Stone, 1974). La valeur du RQMS du modèle composite 1 (qui ne comprend pas la variable modératrice des croyances relatives à la législation) est de 0,111 , ce qui est légèrement proche de la valeur 0,10 recommandée par Bentler (1995), ce qui indique un bon ajustement du modèle.
Le pouvoir explicatif des modèles d'équations structurelles a été évalué à l'aide du coefficient de détermination $\mathrm{R}^{2}$, qui évalue la capacité du modèle à prédire les construits endogènes. La valeur $\mathrm{du} \mathrm{R}^{2}$ ajusté était de 0,552, ce qui implique que 55,2\% de la variance de l'intention de déconsommation d'électricité s'explique par des variables de démarketing, soit une proportion relativement bonne en sciences sociales.

La valeur du RQMS du modèle composite 2 (qui comprend la variable modératrice des croyances relatives à la législation) est de 0,107 . La valeur du $\mathrm{R}^{2}$ ajusté était de 0,585 , ce qui signifie que 58,5\% de la variance dans la décision des consommateurs s'explique par les variables indépendantes considérant l'effet modérateur des croyances relatives à la législation.

De plus, le signe, l'amplitude et la signification des coefficients ont été pris en compte. Une procédure de bootstrapping basée sur 5000 rééchantillons tirés avec remise a été utilisée pour déterminer l'importance de chaque chemin estimé. 


\begin{tabular}{|c|c|c|c|c|c|}
\hline \multirow{2}{*}{$\begin{array}{l}\text { Modèle } \\
\text { ( } n \text { et indices d'ajustement) }\end{array}$} & \multirow{2}{*}{$\begin{array}{l}\text { Variable dépendante : } \\
\text { démarketing }\end{array}$} & \multicolumn{2}{|c|}{ Modèle 1} & \multicolumn{2}{|c|}{ Modèle 2} \\
\hline & & Coef. & Valeur $\mathrm{t}$ & Coef. & Valeur $\mathrm{t}$ \\
\hline \multirow{4}{*}{$\begin{array}{l}\text { Modèle } 1: \text { Modèle de base } \\
n=327 \\
d f=325 \\
\text { RQMS }=0,103 \\
\text { d_ULS }=3,845 \\
\text { d_G }=1,264 \\
\text { khi-deux }=2128,217 \\
\text { IAN }=0,912\end{array}$} & \begin{tabular}{|l|l|} 
& P1
\end{tabular} & 0,171 & $2,170^{*}$ & 0,118 & $1,981 *$ \\
\hline & P2 & 0,237 & $3,544 * * *$ & 0,221 & $3,484^{* * *}$ \\
\hline & P3 & 0,301 & $4,615^{* * *}$ & 0,279 & $3,451 * * *$ \\
\hline & P4 & 0,332 & 5,699 *** & 0,327 & $5,706^{* * *}$ \\
\hline \multirow{5}{*}{$\begin{array}{l}\text { Modèle } 2: \text { Modèle à interac- } \\
\text { tion impliquant les croyances } \\
\text { relatives à la législation } \\
n=327 \\
\text { df }=325 \\
\text { RQMS }=0,096 \\
\text { d_ULS }=4,954 \\
\text { d_G }=1,548 \\
\text { khi-deux }=2643,342 \\
\text { IAN }=0,926\end{array}$} & CRL & & & 0,154 & $1,996^{*}$ \\
\hline & $\mathrm{P} 1 \times \mathrm{CRL}$ & & & 0,065 & 1,360 \\
\hline & $\mathrm{P} 2 \times \mathrm{CRL}$ & & & 0,157 & $2,608^{* *}$ \\
\hline & P3 $\times$ CRL & & & 0,145 & $2,274^{*}$ \\
\hline & $\mathrm{P} 4 \times \mathrm{CRL}$ & & & 0,093 & $1,997^{*}$ \\
\hline
\end{tabular}

Tableau 5 - Résultats des modèles d'équations structurelles

Les résultats de l'étude indiquent des relations positives entre les intentions de déconsommation d'électricité et la perception de stratégies de démarketing liées au produit $(\beta=0,096 ; \mathrm{t}=2,240$; $p<0,05)$, au prix $(\beta=0,279 ; \mathrm{t}=4,554 ; p<0,0000)$, au lieu $(\beta=0,351 ; \mathrm{t}=5,484 ; p<0,000)$ et à la promotion $(\beta=0,295 ; \mathrm{t}=5,744 ; p<0,000)$. Ces résultats valident collectivement les hypothèses 1 à 4 (H1-H4).

Nous avons également émis l'hypothèse selon laquelle ces quatre relations seraient modérées par les croyances relatives à la législation. Les résultats du tableau 5 appuient en partie les hypothèses proposées $(\mathrm{H} 5 \mathrm{a}-\mathrm{H} 5 \mathrm{~d})$, qui prédisaient que la relation respective entre les intentions de déconsommation des consommateurs et le produit, le prix, le lieu et la promotion serait renforcée par les croyances relatives à la législation. Les données soutiennent H5b (variable prix; $\beta=0,206 ; t=3,634 ; p<0,000$ ), $\mathrm{H} 5 \mathrm{c}$ (variable lieu; $\beta=0,128 ; \mathrm{t}=2,068 ; p<0,05$ ) et $\mathrm{H} 5 \mathrm{~d}$ (variable promotion; $\beta=0,097$; $\mathrm{t}=2,089 ; p$ $<0,05$ ), mais pas H5a (variable produit; $\beta=0,036$; $\mathrm{t}=1,042, \mathrm{~ns})$.

\section{Le genre}

Concernant les variables de contrôle, les résultats des tests d'échantillons indépendants indiquent que les femmes et les jeunes consommateurs sont plus susceptibles d'être affectés par les éléments de démarketing $[\mathrm{F}=0,163 ; \mathrm{t}=3,46 ; p=0,000]$. Ces résultats sont en accord avec Sakah et ses collègues (2019) et Aslam et Ahmad (2018), qui ont constaté que le genre a un effet positif et statistiquement pertinent sur la consommation d'électricité. L'utilisation de l'électricité par les ménages révèle que les ménages dirigés par un homme ont tendance à utiliser plus d'énergie en moyenne que les ménages dirigés par des femmes, ce qui confirme les études précédentes sur les différences entre les genres dans l'action responsable et proenvironnementale (Ertz et collab., 2016).

Comme nous l'avons vérifié par les tests de la plus petite différence significative (least significant difference test ou LSD) post-hoc qui ont suivi l'analyse de la variance, l'impact de la stratégie de démarketing perçue sur la consommation d'électricité est substantiellement différent pour divers groupes démographiques, précisément l'âge $(\phi<0,034)$, le niveau d'éducation $(p<0,018)$, l'état matrimonial $(\phi<0,027)$ et le revenu mensuel $(p<0,013)$. 


\section{L'âge}

Le test post-hoc révèle que les personnes de 21 à 30 ans sont plus susceptibles d'être affectées par les stratégies de démarketing que les personnes de 51 à 60 ans. Cela peut être dû au fait que les consommateurs plus âgés passent plus de temps à la maison, ce qui nécessite plus de systèmes de chauffage et de climatisation (Huang, 2015). Selon Aslam et Ahmad (2018), les économistes ont récemment reconnu l'intérêt d'étudier le comportement de consommation d'énergie de différentes tranches d'âge, notamment dans le contexte des changements climatiques. À cet égard, la littérature connexe affirme que le vieillissement de la population déclenchera le ralentissement des tentatives des décideurs politiques de réduire l'intensité énergétique (Harper, 2013), ce qui est cohérent avec des études (p. ex., Xu et collab., 2020; Huebner et collab., 2016) qui montrent l'importance de l'âge dans la conservation de l'énergie.

\section{Le niveau d'éducation}

Le test post-hoc révèle également que les personnes ayant un diplôme d'études secondaires ou inférieur sont plus susceptibles d'être influencées par des stratégies de démarketing visant à réduire la consommation d'électricité que les personnes titulaires d'un baccalauréat ou d'une maitrise. De plus, des différences statistiquement significatives existent entre les titulaires d'un diplôme et les titulaires d'une maitrise, ce qui favorise les titulaires d'un diplôme. Ces différences pourraient s'expliquer en partie par une plus grande utilisation d'énergie par les personnes plus instruites pour maintenir leur mode de vie (Aslam et Ahmad, 2018; Miah et collab., 2011), conformément aux études d'Azlina et ses collègues (2016) et de Zhou et Teng (2013).

Bien que l'éducation puisse sembler préjudiciable à la déconsommation d'énergie, il convient de noter qu'elle est également un moteur clé de la croissance économique et de l'innovation. Elle pourrait donc être considérée comme un des outils dont disposent les pays pour promouvoir l'éducation à l'énergie, la mise en œuvre des énergies renouvelables et la consommation réduite d'énergie (Inglesi-Lotz et Morales, 2017). En conséquence, les principes de conservation de l'énergie et de déconsommation devraient être intégrés dans les programmes d'enseignement.

\section{L'état matrimonial}

Le test post-hoc confirme que les personnes mariées sont plus susceptibles que les célibataires d'être influencées par les stratégies de démarketing. Cela pourrait être dû au fait que la vie des personnes mariées est plus susceptible d'être stable. Cependant, la littérature rapporte des résultats contradictoires : Azlina et ses collègues (2016) établissent l'état matrimonial comme un des facteurs qui influencent la consommation d'énergie, tandis que Sardianou (2007) considère que l'état matrimonial n'a pas de relation significative avec la consommation d'énergie.

\section{Le revenu mensuel}

Le test post-hoc révèle que les personnes ayant un revenu mensuel inférieur $(0 \$$ à $1000 \$)$ sont plus susceptibles d'être influencées par les stratégies de démarketing que celles ayant un revenu mensuel plus élevé (1001\$ à $1500 \$)$. Il peut y avoir plusieurs raisons à cela. Puisque leur revenu détermine leur pouvoir d'achat, un revenu plus élevé leur permet de consommer plus d'électricité que les personnes à revenu plus faible (Zhang et collab., 2017; Su, 2018).

Selon Yalcintas et Kaya (2017), la consommation d'électricité peut augmenter à mesure que le prix de l'électricité augmente, si les revenus augmentent au même taux ou plus. Dans la lignée de Sun et Lin (2013), Zou et ses collègues (2020) montrent que l'élasticité-prix de la demande d'énergie varie selon les groupes de revenus.

Ainsi, les groupes à faible revenu font face à des contraintes financières plus importantes, de sorte que les changements d'éléments de la stratégie de démarketing, en particulier le prix, mais aussi l'accès à l'électricité et la perception du produit, ont un impact plus important sur leur demande en électricité. Ils sont plus susceptibles de répondre aux campagnes incitant à la déconsommation d'électricité, car celle-ci est plus en phase avec leur mode de vie. Quant aux groupes à revenu élevé, ils possèdent plus d'appareils énergivores et une plus grande dépendance à l'électricité.

Ces causes sont cohérentes avec les conclusions de Lee et ses collègues (2020), qui suggèrent que le revenu est le principal facteur de la hausse de la 
consommation d'électricité résidentielle dans les pays en développement.

\section{Discussion}

Après un examen détaillé de l'analyse statistique des données, les principaux résultats sont discutés, et des informations particulières sont fournies pour des pistes de recherche. L'étude a examiné si les intentions de consommation des consommateurs peuvent être réduites en utilisant des stratégies de démarketing. Par ailleurs, l'étude teste également l'efficacité de la réglementation à influencer les intentions de déconsommation des consommateurs.

Les résultats montrent des relations positives entre les variables indépendantes (produit, prix, lieu et promotion) et la variable dépendante (intentions de déconsommation d'électricité). Ces résultats reproduisent ceux obtenus par Salem et ses collègues (2021) dans la BdG. Les résultats indiquent que les stratégies de démarketing sur (par ordre d'importance) le lieu, la promotion, le prix et le produit augmentent les intentions de déconsommation des consommateurs.

D'abord, les stratégies de démarketing liées au lieu (p. ex., la proposition de solutions plus justes comme la prévention des connexions illégales, une distribution équitable entre les emplacements, des importations accrues et des réseaux énergétiques plus efficaces) jouent le rôle le plus crucial dans la déconsommation d'électricité.

Les stratégies de démarketing par la promotion viennent en seconde position. Ainsi, les campagnes de sensibilisation à la baisse de la consommation d'électricité exercent un effet important - mais inférieur aux modulations de la distribution - sur le comportent effectif de déconsommation.

La modulation de prix en fonction du niveau de consommation est la troisième stratégie de démarketing ayant le plus fort impact sur les intentions de déconsommation. Enfin, le produit est une stratégie de démarketing relativement moins efficace.

Cependant, et surtout, contrairement aux recherches antérieures, nous avons émis l'hypothèse selon laquelle les croyances relatives à la législation modéreraient ces quatre relations. En d'autres termes, alors que les croyances relatives à la législation modèrent la relation entre trois des variables de la combinaison démarketing (le prix, le lieu et la promotion) et les intentions de déconsommation, elles ne modèrent pas la relation entre le produit et les intentions de déconsommation.

Les résultats indiquent que la croyance en une législation plus stricte n'est peut-être pas un moyen efficace de réduire directement les intentions de consommation. Au lieu de cela, les consommateurs sont plus susceptibles de réduire leur consommation s'ils :

1. se voient proposer une tarification dynamique basée sur leur niveau de consommation (prix);

2. ont accès à un réseau plus efficace/équitable (lieu); et

3. sont exposés à des campagnes promotionnelles pour la réduction de la consommation d'électricité (promotion).

Ces résultats complètent l'appel de Little et ses collègues (2019) à un «macrodémarketing», qui implique d'aller au-delà des stratégies de gestion et de considérer des instruments plus larges (p. ex., les institutions, la législation et les outils politiques) pour changer les systèmes de production et de consommation non durables.

L'effet positif constant du lieu et du produit sur le démarketing dans le cadre d'une réglementation plus stricte visant à réduire la consommation d'électricité est intuitif. Cependant, une enquête plus approfondie sur ces relations dévoile quelques éléments encourageants. Il semble que les stratégies de démarketing sur les variables du prix, du lieu et de la promotion contribueraient aux intentions réelles de déconsommation d'électricité. D’un point de vue pratique, cela incite les fournisseurs privés à modifier les prix, la chaîne d'approvisionnement et les stratégies de communication, sans nécessairement changer la nature du produit. Ces résultats devraient encourager les autorités à formuler une législation soutenant les efforts des entreprises pour un impact accru sur les intentions de déconsommation.

En plus de confirmer la recherche précédente de Kim et Shin (2016) qui concluait que les modulations de prix conduisent au démarketing, notre étude s'étend à la découverte de deux éléments supplémentaires : le lieu et la promotion. De plus, la réponse des consommateurs à la réglementation gouvernementale peut augmenter l'efficacité du démarketing. 
Dans l'ensemble, les résultats de notre étude confirment l'hypothèse de White et Thomas (2016) selon laquelle le prix et le lieu influencent les intentions de déconsommation. Nous constatons que la promotion et le produit les influencent également. Bien que notre étude porte sur un produit immatériel ('électricité), tandis que celle de White et Thomas (2016) porte sur un produit tangible (les cigarettes), les résultats sont partiellement similaires. Ainsi, il peut apparaittre que, pour les services publics, comme pour les autres biens immatériels, les modulations de produits perçus sont aussi propices à la déconsommation que dans le cas des biens tangibles. Cependant, l'effet produit n'est pas significativement renforcé dans le cadre d'une législation accrue soutenant le démarketing. Par conséquent, les modulations de produits des fournisseurs à elles seules peuvent influencer la déconsommation sans le soutien des décideurs politiques. Or, l'impact des modulations par les trois autres éléments de la combinaison démarketing (prix, lieu et promotion) est renforcé par l'impact de la réglementation.

Contrairement aux recherches antérieures sur le tabagisme - qui ne prenaient pas en compte la promotion, mais se concentraient uniquement sur le produit, le prix et le lieu (p. ex., White et Thomas, 2016) -, nous montrons que la promotion joue un rôle important dans le démarketing. Cependant, elle est moins renforcée positivement avec le soutien de la législation, tandis que le prix et le lieu sont plus fortement renforcés sous l'effet de la perception d'une législation plus stricte.

La modulation des prix semble la variable de démarketing qui interagit le plus fortement avec la réglementation qui l'accompagne, ce qui indique que la modulation des prix (p. ex., la tarification dynamique) contribuerait plus efficacement à la déconsommation d'électricité dans le cadre d'une législation plus stricte. Nous sommes d'accord avec l'accent mis par Link et Stewart-Ladewig (2005) sur l'importance de la tarification pour atteindre les résultats sociétaux souhaitables, qui, dans notre cas, est la déconsommation d'électricité pour une utilisation rationalisée et plus durable.

\subsection{Limites et avenues de recherche}

Puisque notre étude est géographiquement limitée à la $\mathrm{BdG}$, ses résultats ne peuvent pas être généralisés pour toutes les régions de la Palestine, y compris la Cisjordanie. Des recherches pourraient évaluer les facteurs de démarketing pour rationaliser l'électricité dans les deux territoires palestiniens à des fins de comparaison. Israël connait également une pénurie d'électricité; des études - dans la lignée de Grinstein et Nisan (2009) - pourraient approfondir les leviers d'action pour pallier la situation dans ce contexte spécifique.

La collecte de données dans l'étude a utilisé des données autodéclarées. Par conséquent, il est recommandé que des recherches supplémentaires incluent plusieurs méthodes de collecte de données, diverses mesures et une triangulation des données pour évaluer plus précisément les variables de démarketing de la consommation d'électricité. Notre étude était également basée sur la collecte de données transversales; des recherches pourraient mener une étude longitudinale pour évaluer l'impact des stratégies de démarketing sur la consommation d'électricité.

Il serait également fructueux de poursuivre les recherches pour étudier les facteurs affectant la décommercialisation de la consommation d'électricité pour chaque secteur (résidentiel, industriel, agricole, etc.). Un tel examen plus approfondi aiderait à la mise en place de stratégies de déconsommation sectorielles.

Enfin, il existe des différences culturelles entre la société palestinienne et les communautés occidentales. Considérer de tels paramètres spécifiques à la culture comme modérateur sur le lien entre démarketing et déconsommation des consommateurs peut être une piste intéressante pour de futures recherches.

\section{REMERCIEMENTS}

Cette recherche a été menée dans le cadre du programme "Pont Québec-Palestine », financé par le Fonds de Recherche du Québec (FRQ) et coordonnépar la Palestine Academy for Science and Technology (PALAST). La recherche a été menée au Collège universitaire de sciences appliquées de Gaza, à l'Université du Québec à Chicoutimi, an Canada, et à l'Université MoGill, an Canada. 


\section{NOTES}

1 Cet article a été écrit en collaboration avec Myriam Ertz, professeure à l'Université du Québec à Chicoutimi ainsi que Emine Sarigöllü, professeure de marketing à l'Université McGill.

2 Nous nous inspirons de l'expression mix marketing, dont l'équivalent français est combinaison marketing.

3 En anglais, on les surnomme les $4 \mathrm{P}$ : product, price, promotion et place.

\section{RÉFÉRENCES}

Afful-Dadzie, A., Afful-Dadzie, E., Abbey, N. A., Owusu, B. A. et Awudu, I. (2020). Renewable electricity generation target setting in developing countries: Modeling, policy, and analysis. Energy for Sustainable Development, 59, 83-96. https://doi.org/10.1016/j.esd.2020.09.003

Ajzen, I. (1991). The theory of planned behavior. Organizational Behavior and Human Decision Processes, 50(2), 179-211. https://doi.org/10.1016/0749-5978(91)90020-T

Al-Samydai, M. J. et Yousif, R. O. (2018). The role of demarketing in reducing electricity demand. International Journal of Business and Management, 13(1), 209-217. https://doi.org/10.5539/ijbm.v13n1p209

Alvi, F., Prasad, A. et Segarra, P. (2019). The political embeddedness of entrepreneurship in extreme contexts: The case of the West Bank. Journal of Business Ethics, 157, 279-292. https://doi.org/10.1007/s10551-017-3637-9

Andrews, J. C., Netemeyer, R. G., Burton, S., Moberg, D. P. et Christiansen, A. (2004). Understanding adolescent intentions to smoke: An examination of relationships among social influence, prior trial behavior, and antitobacco campaign advertising. Journal of Marketing, 68(3), 110-123. https://doi.org/10.1509/jmkg.68.3.110.34767

Aslam, M. et Ahmad, E. (2018). Impact of ageing and generational effects on household energy consumption behavior: evidence from Pakistan. Energies, 11(8), 2003. https://doi.org/10.3390/en11082003

Ayres, I. et Braithwaite, J. (1992). Responsive regulation: Transcending the deregulation debate. Oxford University Press.

Azlina, A. A., Kamaludin, M., Abdullah, E. S. Z. E. et Radam, A. (2016). Factors influencing household end-use electricity demand in Malaysia. Advanced Science Letters, 22(12), 4120-4123. doi:10.1166/asl.2016.8189

Beeton, S. et Benfield, R. (2002). Demand control: The case for demarketing as a visitor and environmental management tool. Journal of Sustainable Tourism, 10(6), 497-513. https://doi.org/10.1080/09669580208667184

Beeton, S. et Pinge, I. (2003). Casting the holiday dice: Demarketing gambling to encourage local tourism. Current Issues in Tourism, 6(4), 309-322. https://doi.org/10.1080/13683500308667958

Bentler, P. M. (1995). EQS structural equations program manual (version 6) [logiciel]. Encino, CA: Multivariate Software.

Berry, L., Seiders, K. et Grewal, D. (2002). Understanding service convenience. Journal of Marketing, 66(3), 1-17. https://doi.org/10.1509/jmkg.66.3.1.18505

Black, J. (2002). Critical reflections on regulation. Australasian Journal of Legal Philosophy, 27(1), 1-35. https://search.informit.org/doi/10.3316/ielapa.200206927

Bradley, N. et Blythe, J. (2013). Demarketing: An overview of the antecedents and current status of the discipline. Dans N. Bradley et J. Blythe (dir.), Demarketing (p. 17-23). Routledge.

Chin, W. (1998). Issues and opinion on structural equation modeling. MIS Quarterly, 22(1), 7-16. https://www.jstor.org/stable/249674

Craig, C. et Feng, S. (2017). Exploring utility organization electricity generation, residential electricity consumption, and energy efficiency: A climatic approach. Applied Energy, 185(1), 779-790. https://doi.org/10.1016/j.apenergy.2016.10.101

Doggart, N., Ruhinduka, R., Meshack, C. K., Ishengoma, R. C., Morgan-Brown, T., Abdallah, J. M., Spracklen, D. V. et Sallu, S. M. (2020). The influence of energy policy on charcoal consumption in urban households in Tanzania. Energy for Sustainable Development, 57, 200-213. https://doi.org/10.1016/j.esd.2020.06.002

Donoghue, S. et de Klerk, H. M. (2006). Dissatisfied consumers' complaint behaviour concerning product failure of major electrical household appliances: A conceptual framework. Journal of Family Ecology and Consumer Sciences, 34, 41-55. https://repository.up.ac.za/bitstream/handle/2263/2843/Donoghue_Dissatisfied(2006).pdf?sequence=1 
Dua, S., Pise, A., Sreedhar, D., Manthan, J., Ligade, V. et Udupa, N. (2007). Demarketing in healthcare: Concept and approaches. Pharma Review, 212.

Ertz, M., Karakas, F. et Sarigöllü, E. (2016). Exploring pro-environmental behaviors of consumers: An analysis of contextual factors, attitude, and behaviors. Journal of Business Research, 69(10), 3971-3980. https://doi.org/10.1016/j.jbusres.2016.06.010

Fornell, C. et Larcker, D. F. (1981). Evaluating structural equation models with unobservable variables and measurement error. Journal of Marketing Research, 18(1), 39-50. https://doi.org/10.1177/002224378101800104

Geisser, S. (1975). The predictive sample reuse method with applications. Journal of the American Statistical Association, 70(350), $320-328$. https://www.tandfonline.com/doi/citedby/10.1080/01621459.1975.10479865?scroll=top\&needAccess=true

Gerstner, E., Hess, J. et Chu, W. (1993). Demarketing as a differentiation strategy. Marketing Letters, 4(1), 49-57. https://doi.org/10.1007/BF00994187

Grinstein, A. et Nisan, U. (2009). Demarketing, minorities, and national attachment. Journal of Marketing, 73(2), 105-122. https://doi.org/10.1509/jmkg.73.2.105

Gutiérrez-Pedrero, M., Tarancón, M., del Río, P. et Alcántara, V. (2018). Analysing the drivers of the intensity of electricity consumption of non-residential sectors in Europe. Applied Energy, 211, 743-754. https://doi.org/10.1016/j.apenergy.2017.10.115

Harper, S. (2013). Population-environment interactions: European migration, population composition and climate change. Environmental and Resource Economics, 55(4), 525-541. https://doi.org/10.1007/s10640-013-9677-4

Henseler, J., Ringle, C. M. et Sinkovics, R. R. (2009). The use of partial least squares path modeling in international marketing. Dans R. Sinkovics et P. Ghauri (dir.), New challenges to international marketing (p. 277-319). Emerald Group Publishing.

Heylen, E., Papadaskalopoulos, D., Konstantelos, I. et Strbac, G. (2020). Dynamic modelling of consumers' inconvenience associated with demand flexibility potentials. Sustainable Energy, Grids and Networks, 21, 100298. https://doi.org/10.1016/j.segan.2019.100298

Huang, W. H. (2015). The determinants of household electricity consumption in Taiwan: Evidence from quantile regression. Energy, 87, 120-133. doi: 10.1016/j.energy.2015.04.101

Huebner, G., Shipworth, D., Hamilton, I., Chalabi, Z. et Oreszczyn, T. (2016). Understanding electricity consumption: A comparative contribution of building factors, socio-demographics, appliances, behaviours and attitudes. Applied energy, 177, 692-702.

Inglesi-Lotz, R. et Morales, L. D. D. C. (2017). The effect of education on a country's energy consumption: evidence from developed and developing countries. ERS A work pap, 678.

Ismail, M., Moghavvemi, M. et Mahlia, T. (2013). Energy trends in Palestinian territories of West Bank and Gaza Strip: Possibilities for reducing the reliance on external energy sources. Renewable and Sustainable Energy Reviews, 28, 117-129. https://doi.org/10.1016/j.rser.2013.07.047

Japan International Cooperation Agency (JICA). (2017). Data collection survey on Gaza reconstruction in water and energy sector in Palestine: Final report (vol. II Energy Sector). https:/ / openjicareport.jica.go.jp/pdf/12301578.pdf

Kern, C. (2006). Demarketing as a tool for managing visitor demand in national parks: An Australian case study [Mémoire de maitrise, University of Canberra]. https://researchsystem.canberra.edu.au/ws/portalfiles/portal/33684442/file

Khadka, K. et Maharjan, S. (2017). Customer satisfaction and customer loyalty [Thèse de doctorat, Centria University of Applied Sciences]. Theseus. https://www.theseus.fi/bitstream/handle/10024/139650/khadka_kabu\%20\%20and\%20\%20maharjan_\%20soniya.pdf?sequence $=1$

Kim, J. et Shin, D. (2016). Price discrimination with demarketing. The Journal of Industrial Economics, 64(4), 773-807. https://doi.org/10.1111/joie.12129

Kotler, P. (2017). My adventures in marketing: The autobiography of Philip Kotler. Idea Bite Press.

Kotler, P. et Levy, S. (1971). Demarketing, yes, demarketing. Harvard Business Review, 49, 74-80. https://web.uniroma1.it/ dip_management/sites/default/files/allegati/Kotler $\% 20 \% 26 \% 20$ Levy $\% 20 \% 281971 \% 29 \% 20-\% 20$ Demarketing $\% 2 \mathrm{C} \% 20$ yes $\% 2 \mathrm{C} \% 20$ demarketing $\% 20-\% 20$ Harvard $\% 20$ Business $\% 20$ Review.pdf

Lancaster, T., Stead, L., Silagy, C. et Sowden, A. (2000). Effectiveness of interventions to help people stop smoking: Findings from the Cochrane Library. British Medical Journal, 321(7257), 355-358. https:/ / doi.org/10.1136/sbmj.0009323 
Lee, C. Y., Kaneko, S. et Sharifi, A. (2020). Effects of building types and materials on household electricity consumption in Indonesia. Sustainable Cities and Society, 54, 101999. doi:10.1016/j.scs.2019.101999

Link, H. et Stewart-Ladewig, L. (2005). Basic road pricing solutions. Research in Transportation Economics, 11, 9-26. https://doi.org/10.1016/S0739-8859(05)80005-8

Little, V. J., Lee, C. K. C. et Nair, S. (2019). Macro-demarketing: The key to unlocking unsustainable production and consumption systems? Journal of Macromarketing, 39(2), 166-187. https://doi.org/10.1177/0276146718823885

Maloney, M. (2014). The role of regulation in reducing consumption by individuals and households in industrialised nations [Thèse de doctorat, Griffith University]. https://research-repository.griffith.edu.au/handle/10072/368147

Maxwell, S. (2002). Rule-based price fairness and its effect on willingness to purchase. Journal of Economic Psychology, 23(2), 191-212. https://doi.org/10.1016/S0167-4870(02)00063-6

Medway, D., Warnaby, G. et Dharni, S. (2011). Demarketing places: Rationales and strategies. Journal of Marketing Management, 27(1-2), 124-142. https://doi.org/10.1080/02672571003719096

Miah, M. D., Foysal, M. A., Koike, M. et Kobayashi, H. (2011). Domestic energy-use pattern by the households: A comparison between rural and semi-urban areas of Noakhali in Bangladesh. Energy Policy, 39(6), 3757-3765. https://EconPapers.repec.org/RePEc:eee:enepol:v:39:y:2011:i:6:p:3757-3765

Ministry of Defense. (2007). Defense Export Control Law, 5766-2007 [traduction non officielle]. Gouvernement de la Palestine. http://www.exportctrl.mod.gov.il/English/Pages/Defense-Export-Control-Law-.aspx

Monfared, H. J. et Ghasemi, A. (2019). Retail electricity pricing based on the value of electricity for consumers. Sustainable Energy, Grids and Networks, 18, 100205. https://doi.org/10.1016/j.segan.2019.100205

Nilsson, A., Stoll, P. et Brandt, N. (2017). Assessing the impact of real-time price visualization on residential electricity consumption, costs, and carbon emissions. Resources, Conservation and Recycling, 124, 152-161. https://doi.org/10.1016/j.resconrec.2015.10.007

Niroomand, N. et Jenkins, G. P. (2020). Estimation of households' and businesses' willingness to pay for improved reliability of electricity supply in Nepal. Energy for Sustainable Development, 55, 201-209. https://doi.org/10.1016/j.esd.2020.02.006

Nunnally, J. C. (1978). Psychometric theory (2e éd.). McGraw-Hill.

Nunnally, J. C. et Bernstein, I. H. (1994). Psychometric theory (3e éd.). McGraw-Hill.

Page, C. et Colby, P. (2003). If only I hadn't smoked: The impact of counterfactual thinking on a smoking-related behavior. Psychology \& Marketing, 20(11), 955-976. https://doi.org/10.1002/mar.10104

Palestinian Central Bureau of Statistics (PCBS). (2018). Preliminary results of the population, housing and establishments census 2017. Gouvernement de la Palestine. http://www.pcbs.gov.ps/portals/_pcbs/PressRelease/Press_En_Preliminary_Results_ Report-en.pdf

Pechmann, C. et Knight, S. (2002). An experimental investigation of the joint effects of advertising and peers on adolescents' beliefs and intentions about cigarette consumption. Journal of Consumer Research, 29(1), 5-19. https://doi.org/10.1086/339918

Ramirez, E., Tajdini, S. et David, M. (2017). The effects of proenvironmental demarketing on consumer attitudes and actual consumption. Journal of Marketing Theory and Practice, 25(3), 291-304. https://doi.org/10.1080/10696679.2017.1311219

Richins, M. (1983). Negative word-of-mouth by dissatisfied consumers: A pilot study. Journal of Marketing, 47(1), 68-78. https://doi.org/10.1177/002224298304700107

Ringle, C., Wende, S. et Becker, J. (2015). SmartPLS 3.0 [logiciel]. Bönningstedt: SmartPLS GmbH.

Salem, M. Z. Y. (2013). Factors affecting the demarketing of breastmilk substitutes in Palestine. Breastfeeding Medicine, 8(3), 302-311. https://doi.org/10.1089/bfm.2012.0120

Salem, M. Z., Baidoun, S. et Almuzaini, R. F. (2018). Water consumption demarketing strategies with reference to the Gaza Strip, Palestine. Polish Journal of Environmental Studies, 27(1), 297-304. https://doi.org/10.15244/pjoes/74300

Salem, M. Z., Ertz, M. et Sarigöllü, E. (2021). Demarketing strategies to rationalize electricity consumption in the Gaza Strip-Palestine. Renewable and Sustainable Energy Reviews, 143, 110956. https://doi.org/10.1016/j.rser.2021.110956 
Sakah, M., du Can, S. D. L. R., Diawuo, F. A., Sedzro, M. D. et Kuhn, C. (2019). A study of appliance ownership and electricity consumption determinants in urban Ghanaian households. Sustainable Cities and Society, 44, 559-581. doi: $10.1016 /$ j.scs.2018.10.019

Saunders, M., Lewis, P. et Thornhill, A. (2016). Research methods for business students. Pearson Education.

Seeletse, S. M. (2016). Demarketing strategy to develop perceived product reputation: Applications in three distinct environments. Problems and Perspectives in Management, 14(4), 230-235. https://doi.org/10.21511/ppm.14(4-1).2016.12

Shiu, E., Hassan, L. M. et Walsh, G. (2009). Demarketing tobacco through governmental policies: The 4Ps revisited. Journal of Business Research, 62(2), 269-278. https://doi.org/10.1016/j.jbusres.2008.01.034

Siegel, M. et Biener, L. (2000). The impact of an antismoking media campaign on progression to established smoking: Results of a longitudinal youth study. American Journal of Public Health, 90, 380-386. https://doi.org/10.2105/ajph.90.3.380

Silagy, C. et Stead, L. (2001). Physician advice for smoking cessation. Cocbrane Database of Systematic Reviews, 2, CD000165. https://doi.org/10.1002/14651858.cd000165

Sovacool, B. (2017). Reviewing, reforming, and rethinking global energy subsidies: Towards a political economy research agenda. Ecological Economics, 135, 150-163. https://doi.org/10.1016/j.ecolecon.2016.12.009

Stone, M. (1974). Cross-validatory choice and assessment of statistical predictions. Journal of the Royal Statistical Society: Series B (Methodological), 36(2), 111-133. https://doi.org/10.1111/j.2517-6161.1974.tb00994.x

Su, Y. W. (2018). Electricity demand in industrial and service sectors in Taiwan. Energy Efficiency, 11(6), 1541-1557. doi:10.1007/s12053-018-9615-y

Tesfamichael, M., Bastille, C. et Leach, M. (2020). Eager to connect, cautious to consume: An integrated view of the drivers and motivations for electricity consumption among rural households in Kenya. Energy Research \& Social Science, 63, 101394. https://doi.org/10.1016/j.erss.2019.101394

UN Special Coordinator for the Middle East Peace Process (UNSCO). (2017). Report to the Ad Hoc Liaison Committee. United Nations. https://unsco.unmissions.org/sites/default/files/unsco_report_to_ahlc_-_1_may_2017.pdf

United Nations Country Team (UNCT) in the occupied Palestinian territory. (2017). Gaza ten years later. United Nations. https://unsco.unmissions.org/sites/default/files/gaza_10_years_later_-_11_july_2017.pdf

United Nations Office for the Coordination of Humanitarian Affairs (OCHA). (2020). Gaza Strip electricity supply 2020. https://www.ochaopt.org/page/gaza-strip-electricity-supply

Vandenbergh, M. P. (2004). Order without social norms: How personal norm activation can protect the environment. Northwestern University Law Review, 99(3), 1101-1166. https:// scholarship.law.vanderbilt.edu/cgi/viewcontent.coi?article=2033\&context= faculty-publications

Wall, A. P. (2005). Government demarketing: Different approaches and mixed messages. European Journal of Marketing, 39(5/6), 421-427. https://doi.org/10.1108/03090560510590647

Wall, A. P. (2007). Government “demarketing” as viewed by its target audience. Marketing Intelligence \& Planning, 25(2), 123-135. https://doi.org/10.1108/02634500710737915

White, C. J. et Thomas, H. (2016). Up in smoke: Demarketing and consumer smoking cessation. International Journal of Consumer Studies, 40(1), 75-82. https://doi.org/10.1111/ijcs.12216

Xia, L., Monroe, K. et Cox, J. (2004). The price is unfair! A conceptual framework of price fairness perceptions. Journal of Marketing, 68(4), 1-15. https://doi.org/10.1509/jmkg.68.4.1.42733

Xu, X., Xiao, B. et Li, C. Z. (2020). Critical factors of electricity consumption in residential buildings: An analysis from the point of occupant characteristics view. Journal of Cleaner Production, 256, 120423. doi:10.1016/j.jclepro.2020.120423

Yalcintas, M. et Kaya, A. (2017). Roles of income, price and household size on residential electricity consumption: Comparison of Hawaii with similar climate zone states. Energy Reports, 3, 109-118. https://doi.org/10.1016/j.egyr.2017.07.002

Yousif, R. (2014). Measuring the effectiveness of demarketing in influencing consumer behavior of individuals. International Journal of Business Management \& Research, 4(5), 31-44. 
Zhang, C., Zhou, K., Yang, S. et Shao, Z. (2017). On electricity consumption and economic growth in China. Renewable and Sustainable Energy Reviews, 76, 353-368. https://doi.org/10.1016/j.rser.2017.03.071

Zhou, S. et Teng, F. (2013). Estimation of urban residential electricity demand in China using household survey data. Energy Policy, 61, 394-402. doi: 10.1016/j.enpol.2013.06.092

Zou, H., Luan, B., Zheng, X. et Huang, J. (2020). The effect of increasing block pricing on urban households' electricity consumption: Evidence from difference-in-differences models. Journal of Cleaner Production, 257, 120498. doi: 10.1016/j.jclepro.2020.120498 